\title{
Safe trabeculectomy technique: long term outcome
}

\author{
I Stalmans, A Gillis, A-S Lafaut, T Zeyen
}

Br J Ophthalmol 2006;90:44-47. doi: 10.1136/bjo.2005.072884

See end of article for authors' affiliations

....................

Correspondence to: T Zeyen, MD, PhD, Ophthalmology Department, University Hospital, Kapuciinenvoer 33, B-3000, Leuven, Belgium; thierry.zeyen@ uz.kuleuven.ac.be

Accepted for publication 8 August 2005

\begin{abstract}
Aim: To assess the long term outcome of a new trabeculectomy technique.
Methods: Trabeculectomy was performed using a fornix based conjunctival flap, an anterior chamber maintainer, a standardised punch technique, and a combination of adjustable and releasable sutures in 56 eyes of 53 patients. The main outcome measures were the postoperative intraocular pressure (IOP) and the frequency of early postoperative complications. The mean follow up time was 15.7 (range 12-21) months.

Results: The mean preoperative and postoperative IOP at 12 months were 21.2 (SD 6) and 12.8 (3.0) $\mathrm{mm} \mathrm{Hg}$, respectively. All patients had an IOP of $<21 \mathrm{~mm} \mathrm{Hg}, 90.9 \%$ had an IOP $<18 \mathrm{~mm} \mathrm{Hg}$, and $61.4 \%$ had an IOP $<14 \mathrm{~mm} \mathrm{Hg}$. Postoperative complications were infrequent: flat anterior chamber (1.8\%), bleb leakage $(0 \%)$, or hypotony $(1.5 \%)$ beyond 3 weeks, or choroidal detachment at any time point $(8.9 \%)$. Conclusions: This novel trabeculectomy method offers the possibility to tailor the IOP postoperatively with a minimum of postoperative complications and excellent IOP control at the long term follow up.
\end{abstract}

$\mathrm{T}$ he classic trabeculectomy technique using a rectangular scleral flap with two releasable sutures at the edges and viscoelastic to maintain the anterior chamber (AC) is most routinely used. ${ }^{1}$ However, this technique has three weak points. Firstly, the amount of filtration is variable. Hyperfiltration can result in a flat AC, hypotony maculopathy, choroidal detachment or haemorrhage, aqueous misdirection or cataract. ${ }^{2}$ On the other hand, ocular massage and/or laser suture lysis are frequently necessary to obtain a low postoperative intraocular pressure (IOP). Laser suture lysis is non-titratable, sometimes impossible to perform, and can result in subsequent overdrainage and hypotony. Secondly, peroperative flat chamber and hypotony during trabeculectomy augment the risk of vision threatening complications such as choroidal haemorrhage, macular wipeout, and corneal decompensation. Thirdly, debris that remains at the trabeculectomy site after surgery induces an inflammatory response that induces fibrosis.

A new trabeculectomy technique was developed by Peng Khaw $^{3}$ that implies the use of releasable and adjustable sutures, an AC maintainer, and a punch. This technique has several advantages. Firstly, the punch produces a standard trabeculectomy aperture, and postoperative adjustment of the sutures allows titration of filtration, resulting in a more controlled amount of outflow. Secondly, during the operation, the AC is maintained and hypotony is prevented. Thirdly, inflammatory factors are flushed away from the site of surgery, reducing the risk of bleb failure. Overall, this novel technique may result in a better and safer outcome after filtration surgery. This study was set up to evaluate the outcome and safety of this novel trabeculectomy procedure.

\section{METHODS}

\section{Study design and patient inclusion}

In this retrospective study, the files of patients who underwent trabeculectomy using the described technique between February and December 2003 were reviewed. Patients with a history of preceding filtering surgery were excluded. The main outcome measures were the postoperative IOP and the frequency of early postoperative complications. IOP was recorded on days $1-3$, weeks $1-4$, at 3 months after surgery, and 3 monthly thereafter.

\section{Trabeculectomy technique and postoperative management}

A corneal traction suture was placed with a silk $8 / 0$ and a fornix based conjunctival flap was dissected. Antimetabolites were used when indicated. Gentle diathermy was performed. A scleral flap of $6 \times 4 \mathrm{~mm}$ with side incisions at $0.5 \mathrm{~mm}$ from the cornea was delineated with a $30^{\circ}$ blade (Alcon). The flap was dissected half thickness with a crescent knife (Alcon). The central part of the flap was dissected $1 \mathrm{~mm}$ into clear cornea.

Scleral flap sutures (nylon 10/0) were pre-installed: two adjustables laterally (fig 1 ) and two releasables in between. ${ }^{3}$ A corneal paracentesis was made inferotemporally using a knife of $1.2 \mathrm{~mm}$ (Alcon) and the conic $1.3 \mathrm{~mm}$ Blumenthal AC maintainer (BD-Visitec, Franklin Lakes, NJ, USA) was positioned (fig 2) with the bottle of BSS (Alcon) at $30 \mathrm{~cm}$ above the patient's eye. A trabeculectomy was performed using the Khaw punch of $0.5 \mathrm{~mm}$ (Duckworth and Kent, Hertfordshire, UK), followed by an iridectomy. The flap sutures were closed: the two adjustables at the corners of the flap with four throws and the two releasables buried in between. When judged necessary, based on the flow through the AC maintainer, sutures were loosened, removed, tightened, or added. At a bottle height of $30 \mathrm{~cm}$ above the eye, a flow rate of one drop every 3-4 seconds was expected to result in an IOP of $10-15 \mathrm{~mm} \mathrm{Hg} .{ }^{4}$ Finally, the conjunctiva was closed with two nylon 10/0 sutures using a "pursestring" technique.

To reach the target IOP in the following weeks suture adjustments were done according to the needs. The two lateral flap sutures were adjusted using Khaw's transconjunctival adjustable suture control forceps (Duckworth and Kent) or removed, and/or the releasable sutures where cut with the argon laser $(0.5 \mathrm{~W}, 0.1$ seconds, $100 \mu \mathrm{m})$. Cystic encapsulation of the bleb associated with a rise in IOP was treated by needling. ${ }^{5}$ Failing filtering blebs were treated by needling revision ${ }^{6}$ if the scleral flap was visible.

\section{Statistical analysis}

A Student's $t$ test was used to determine statistically significant differences.

Abbreviations: $A C$, anterior chamber; $I O P$, intraocular pressure 

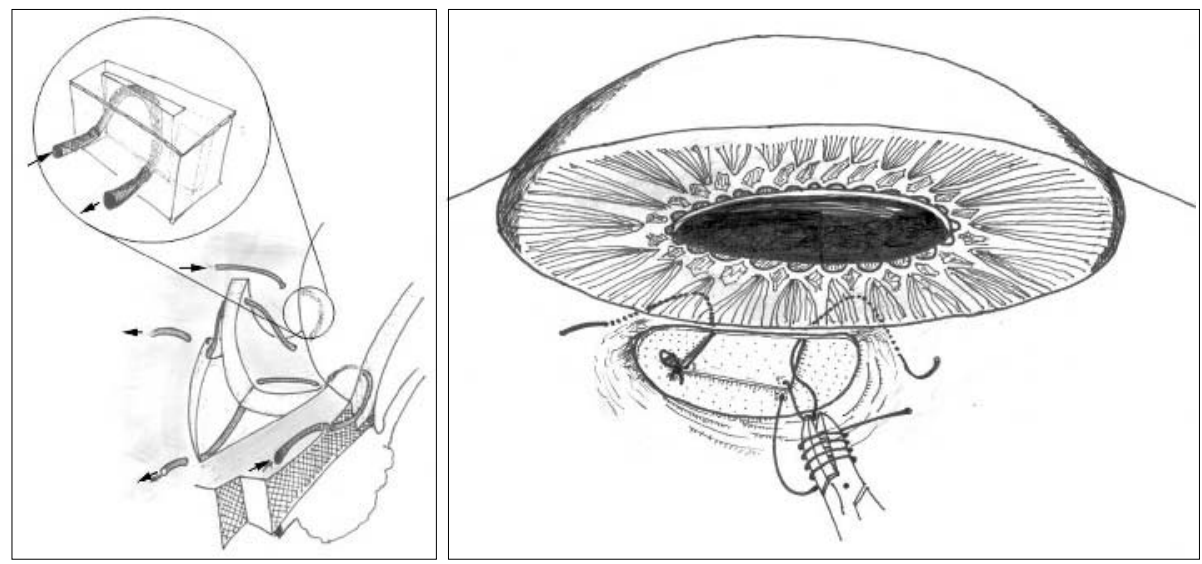

Figure 1 Schematic representation of the adjustable scleral flap sutures. Left: two adjustable sutures are pre-installed at the edges of the scleral flap land two releasable sutures in the middle, not shown), passing through clear cornea (inset), before placing the anterior chamber maintainer. Right: after performing the trabeculectomy and iridectomy, the adjustable flap sutures are closed using four throws.

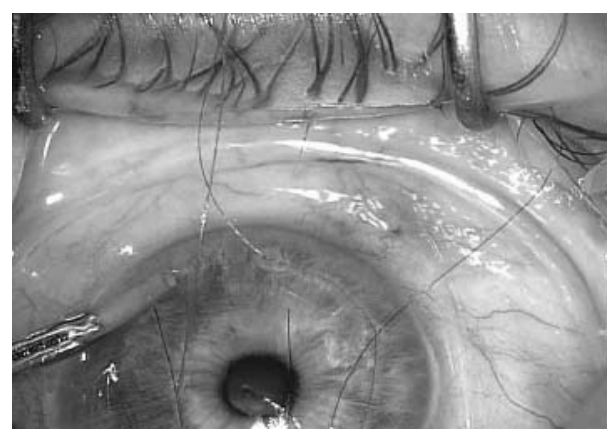

Figure 2 Anterior chamber maintainer. After performing a corneal paracentesis inferotemporally, the anterior chamber infusion is installed with the bottle of BSS at $30 \mathrm{~cm}$ above the patient's eye to maintain the anterior chamber and to constantly flush away any debris or inflammatory factors.

\section{RESULTS}

\section{Patient data}

The baseline characteristics are summarised in tables 1 and 2 .

\section{Trabeculectomy outcome}

The mean follow up time was 15.7 (SD 3.4) months, ranging from 12-21 months. One or two adjustable sutures were removed during surgery in four $(7.1 \%)$ and one $(1.8 \%)$ patients, respectively, and fixed sutures were removed or added in $11(19.6 \%)$ and two (3.6\%) patients, respectively. Mitomycin $\mathrm{C}$ was applied in seven patients (12.5\%) and 5fluorouracil in two patients (3.6\%).

No change in visual acuity was noted: visual acuity before and at 1 month postoperatively was $0.67(0.3)$ (range $0.01-1$ ) and $0.61(0.3)$ (range $0.02-1.0 ; \mathrm{p}=0.25$ ) on average.

The postoperative IOPs are summarised in figure 3 . The day after surgery, the IOP had dropped from $21.2(6.7) \mathrm{mm} \mathrm{Hg}$ to 10.4 (6.2) $\mathrm{mm} \mathrm{Hg}$ (range 2-30). During the first postoperative month, filtration was titrated as summarised in table 3.
These adjustments allowed us to obtain an IOP of 11.8 (4.7) $\mathrm{mm} \mathrm{Hg}$ (range 4-24) at 3 months postoperatively. After this period, the IOP remained stable, with a mean IOP of 12.8 (3.0) $\mathrm{mm} \mathrm{Hg}$ (range 6-19) at 12 months. At this point, all our patients had an IOP of below $21 \mathrm{~mm} \mathrm{Hg}, 90.9 \%$ below $18 \mathrm{~mm} \mathrm{Hg}$, and $61.4 \%$ below $14 \mathrm{~mm} \mathrm{Hg}$ (fig 4). At 15, 18, and 21 months, the mean IOP was 12.8 (3.1), $11.6(3.9)$, and 10.8 (4.0) $\mathrm{mm} \mathrm{Hg}$ in 28, 19, and nine patients, respectively. Four patients $(7.1 \%)$ required IOP lowering medication after trabeculectomy, and needling revision of an inadequately filtrating bleb was necessary once in seven patients $(12.5 \%)$ and twice in three patients $(5.4 \%)$. None of the patients needed a second trabeculectomy (table 4). IOPs were comparable between the patients who underwent trabeculectomy with or without antimetabolites (fig 5).

Postoperative complications are summarised in table 4 .

\section{DISCUSSION}

The adapted technique of trabeculectomy according to Khaw ${ }^{3}$ offers several advantages:

- Controlled filtration: The use of a punch of $0.5 \mathrm{~mm}$ to perform the trabeculectomy results in a consistent opening. The AC maintainer allows for adjusting the flap sutures based on the BSS drip rate. ${ }^{3}$ Postoperatively, filtration can be further titrated by loosening or removing the adjustable sutures, and by laser suture lysis of the releasable sutures.

- Maintenance of AC and pressure: The AC maintainer prevents the AC from collapsing and the pressure from falling abruptly. This results in a reduced frequency of early postoperative complications, such as choroidal detachment and haemorrhage, hypotony maculopathy, corneal decompensation, and cataract formation.

- Reduction of postoperative inflammation and fibrosis: The AC maintainer washes out debris reducing postoperative inflammation (and thus scarring).

Table 1 Baseline characteristics

\begin{tabular}{lll}
\hline Number of patients & 53 & \\
Number of eyes & Left & Right \\
& $21(37.5 \%)$ & $35(62.5 \%)$ \\
Age & Mean (SD) & Range \\
Preoperative IOP* & 65 & $36-85$ \\
Preoperative visual acuity & $21.2(6.7)$ & $10.0-39.6$ \\
Number of preoperative medications & $0.67(0.3)$ & $0.01-1.0$ \\
\hline${ }^{*}$ Mean of the three last measured IOPs. & $2.2(0.9)$ & $1-4$ \\
\hline
\end{tabular}


Table 2 Diagnosis and postocular history

\begin{tabular}{|c|c|c|c|c|c|}
\hline Type of glaucoma & No & $\%$ & Antecedents & No & $\%$ \\
\hline $\begin{array}{l}\text { Primary open angle } \\
\text { Normal tension } \\
\text { Chronic angle closure } \\
\text { Secondary } \\
\text { Juvenile } \\
\text { Ocular hypertension }\end{array}$ & $\begin{array}{r}33 \\
8 \\
9 \\
5 \\
0 \\
1\end{array}$ & $\begin{array}{l}58.9 \\
14.3 \\
16.1 \\
8.9 \\
0.0 \\
1.8\end{array}$ & $\begin{array}{l}\text { Negative } \\
\text { Laser trabeculoplasty } \\
\text { YAG laser iridotomy } \\
\text { Phacoemulsification } \\
\text { Iridotomy, trabeculoplasty } \\
\text { Iridotomy, phaco } \\
\text { Phaco, trabeculoplasty } \\
\text { Intravitreal steroid injection } \\
\text { Acute glaucoma, phaco } \\
\text { Fuchs' heterochromy }\end{array}$ & $\begin{array}{r}29 \\
9 \\
5 \\
2 \\
3 \\
2 \\
2 \\
1 \\
1 \\
1\end{array}$ & $\begin{array}{l}49 \\
15 \\
8.5 \\
3.4 \\
5.1 \\
3.4 \\
3.4 \\
1.7 \\
1.7 \\
1.7\end{array}$ \\
\hline
\end{tabular}

Table 3 Postoperative adjustments and interventions

\begin{tabular}{lrl}
\hline Suture adjustments & No & $\%$ \\
\hline Argon suture lysis of releasable sutures & 21 & 37.5 \\
Loosening of adjustable sutures & 3 & 5.4 \\
Removal of adjustable sutures & 18 & 32.1 \\
Additional surgical intervention & & \\
Needling encapsulated bleb & 8 & 14.3 \\
Needling revision non-functional bleb & 10 & 17.9 \\
Second trabeculectomy & 0 & 0.0 \\
\hline
\end{tabular}

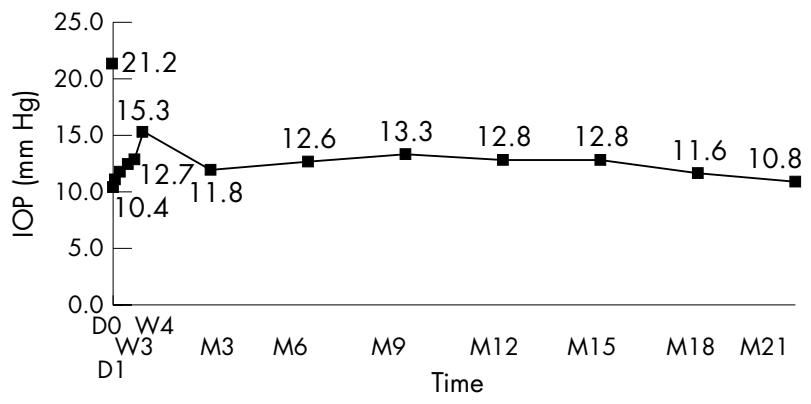

Figure $3 \mathrm{IOP}$ evolution over time. ( $\mathrm{D}=$ day; $M=$ month.)

A fornix based conjunctival flap results in a good visualisation of the surgical field, and allows the aqueous humour to drain posteriorly promoting the formation of a diffuse bleb. ${ }^{7}$ Although a higher incidence of bleb leakage has been reported to be associated with fornix based conjunctival flaps, ${ }^{8}$ the use of "pursestring" technique to close the conjunctiva was efficient in preventing persistent bleb leakage. A wide scleral flap dissected centrally into clear cornea also favours the posterior drainage.

Indeed, mean IOPs of less than $12 \mathrm{~mm} \mathrm{Hg}$ were obtained from the first postoperative day. These pressures remained

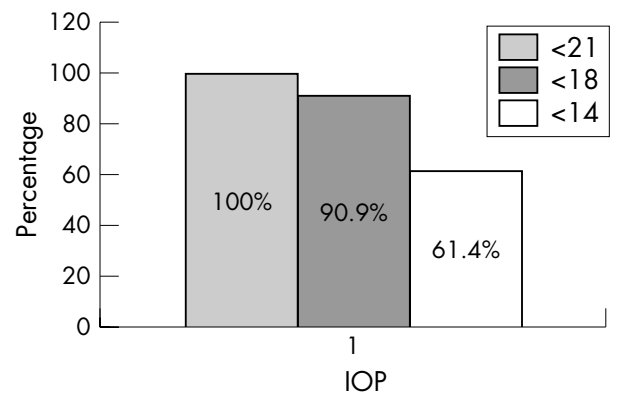

Figure 4 IOP distribution at 12 months.

\section{Table 4 Postoperative course}

\begin{tabular}{lll}
\hline Early complications & No & $\%$ \\
\hline Flat AC requiring surgery & 1 & 1.8 \\
Positive Seidel >3 weeks & 0 & 0.0 \\
Hypotony >3 weeks & 1 & 1.5 \\
Choroidal detachment at any time point & 5 & 8.9 \\
Hyphaema & 1 & 5.4 \\
Corneal epitheliopathy or erosion & 3 & 5.4 \\
Malignant glaucoma & 1 & 1,5 \\
Endophthalmitis & 1 & 1.5 \\
\hline
\end{tabular}

remarkably stable over the follow up period of at least 12 months and up to 21 months: at 12 months postoperatively, the mean IOP was 12.8 (3.0) $\mathrm{mm} \mathrm{Hg}$ (fig 3); all patients had an IOP of $\leqslant 21 \mathrm{~mm} \mathrm{Hg}, 90.9 \%<18 \mathrm{~mm} \mathrm{Hg}$, and $61.4<14 \mathrm{~mm} \mathrm{Hg}$ (fig 4). Of note, there was a transient elevation of the mean IOP $(15.3(5.6) \mathrm{mm} \mathrm{Hg})$ at postoperative week 4 . This peak was explained by the fact that eight patients $(14.3 \%)$ developed an encapsulated bleb. The incidence of bleb encapsulation was low. ${ }^{7}$ After needling of the encapsulated bleb, all patients except one recovered IOPs of below $17 \mathrm{~mm} \mathrm{Hg}$.

Intraoperative complications did not occur, and postoperative complications were infrequent (table 4). However, peroperative and postoperative maintenance of the AC did not prevent malignant glaucoma from occurring in one patient in the immediate postoperative period and in another patient several months after surgery. The use of antiproliferative agents did not influence the final IOP outcome. This was not surprising since antimitotics were used not only in patients with low tension glaucoma, but also in patients who are prone to develop an increased fibrotic reaction, such as those with inflammatory glaucoma or black patients.

These results compare favourably with previously published data using the trabeculectomy technique introduced by Cairns $^{9}$ and modified by Watson. ${ }^{10-21}$ Of note, in the Otago

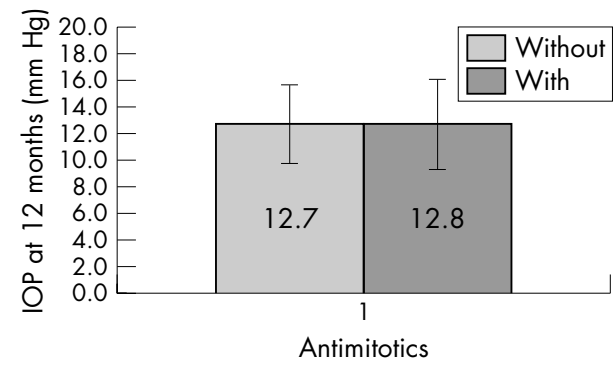

Figure 5 Mean IOP at 12 months without or with use of antimetabolites. 
Glaucoma Surgery Outcome Study, ${ }^{16} 1.8 \%$ of patients were hypotonous $(<6 \mathrm{~mm} \mathrm{Hg})$ at 12 months, whereas this long term hypotony did not occur in our series of patients. Postoperative complications were more frequent than in our series.. ${ }^{11} 12161921$ In the Collaborative Initial Glaucoma Treatment Study, the rate of postoperative complications was $10 \%$, and only $50 \%$ of the patients had a complication free postoperative period. The fact that mean IOPs of $<14 \mathrm{mmHg}$ can result in stable visual fields has been concluded from several major clinical studies. ${ }^{17}{ }^{22}$ Moreover, IOP fluctuations are a known risk factor for visual field progression. ${ }^{23}$ In this respect, the low as well as stable IOPs in our study are a favourable prognostic sign for stable visual function.

Importantly, a significant IOP decrease was also obtained in the normotensive patients, even after 21 months (10.8 (4.0 $\mathrm{mm} \mathrm{Hg}$ ). Previous reports have made it obvious that IOP lowering in normal tension glaucoma is less efficient with medication or laser treatment. ${ }^{24}$

Taken together, these results indicate that this novel trabeculectomy technique is safe, allows postoperative tailoring of the IOP, and results in steadily low IOPs. Prospective, randomised, comparative trials will be needed to further validate this promising trabeculectomy technique.

\section{ACKNOWLEDGEMENTS}

We thank L Vanmalderen for her assistance with the statistical analyses and T Missotten for his artistic help.

\section{Authors' affiliations}

I Stalmans, A-S Lafaut, T Zeyen, Ophthalmology Department, University Hospital, Leuven, B-3000, Belgium

A Gillis, Algemeen Ziekenhuis Middelheim, B-2020 Antwerpen

\section{REFERENCES}

1 Edmunds B, Thompson JR, Salmon JF, et al. The National Survey of Trabeculectomy. II. Variations in operative technique and outcome. Eye $2001 ; 15(\mathrm{Pt} 4): 441-8$

2 Edmunds B, Thompson JR, Salmon JF, et al. The National Survey of

Trabeculectomy. III. Early and late complications. Eye 2002;16:297-303.
3 Wells AP, Bunce C, Khaw PT. Flap and suture manipulation after trabeculectomy with adjustable sutures: titration of flow and intraocular pressure in guarded filtration surgery. J Glaucoma 2004;13:400-6.

4 Papadopoulos M, Khaw PT. Improving glaucoma filtering surgery. Eye 2001; 15(Pt 2):131-2.

5 Feyi-Waboso A, Ejere $\mathrm{HO}$. Needling for encapsulated trabeculectomy filtering blebs. Cochrane Database Syst Rev 2004;(2):CD003658.

6 Broadway DC, Bloom PA, Bunce C, et al. Needle revision of failing and failed trabeculectomy blebs with adjunctive 5-fluorouracil: survival analysis. Ophthalmology 2004;111:665-73.

7 Wells AP, Cordeiro MF, Bunce C, et al. Cystic bleb formation and related complications in limbus- versus fornix-based conjunctival flaps in pediatric and young adult trabeculectomy with mitomycin C. Ophthalmology 2003;110:2192-7.

8 Henderson HW, Ezra E, Murdoch IE. Early postoperative trabeculectomy leakage: incidence, time course, severity, and impact on surgical outcome. Br J Ophthalmol 2004;88:626-9.

9 Cairns JE. Trabeculectomy. Preliminary report of a new method. Am J Ophthalmol 1968;66:673-9.

10 Watson PG. Surgery of the glaucomas. Br J Ophthalmol 1972;56:299-306.

11 Wilson P. Trabeculectomy: long-term follow-up. Br J Ophthalmol 1977;61:535-8.

12 Mills KB. Trabeculectomy: a retrospective long term follow up of 444 cases. Br J Ophthalmol 1981;65:790-5.

13 Kidd MN, O'Connor M. Progression of field loss after trabeculectomy: a 5 year follow up. Br J Ophthalmol 1985;69:827-31.

14 Nouri-Mahdavi K, Brigatti L, Weitzman M, et al. Outcomes of trabeculectomy for primary open-angle glaucoma. Ophthalmology 1995;102:1760-9.

15 Molteno AC, Bosma NJ, Kittelson JM. Otago glaucoma surgery outcome study: long-term results of trabeculectomy-1976 to 1995. Ophthalmology 1999; 106:1742-50.

16 Feiner L, Piltz-Seymour JR. Collaborative Initial Glaucoma Treatment Study: a summary of results to date. Curr Opin Ophthalmol 2003;14:106-11.

17 Migdal C, Gregory W, Hitchings R. Long-term functional outcome after early surgery compared with laser and medicine in open-angle glaucoma. Ophthalmology 1994;101:1651-6 discussion 1657.

18 Jerndal T, Lundstrom M. 330 trabeculectomies - a follow-up study through $1 / 2-3$ years. Acta Ophthalmol (Copenh) 1977;55:52-62.

19 Blondeau P, Phelps CD. Trabeculectomy vs thermosclerostomy. A randomized prospective clinical trial. Arch Ophthalmol 1981;99:810-6.

20 Lewis RA, Phelps CD. Trabeculectomy $v$ thermosclerostomy. A five-year follow-up. Arch Ophthalmol 1984;102:533-6.

21 Lavin MJ, Wormald RP, Migdal CS, et al. The influence of prior therapy on the success of trabeculectomy. Arch Ophthalmol 1990;108:1543-8. 22 The Advanced Glaucoma Intervention Study (AGIS): 7. The relationship AGIS Investigators. Am J Ophthalmol 2000;130:429-40.

23 Nouri-Mahdavi K, Hoffman D, Coleman AL, et al. Predictive factors for glaucomatous visual field progression in the Advanced Glaucoma Intervention Study. Ophthalmology 2004; 111:1627-35.

24 Hoyng PF, Kitazawa Y. Medical treatment of normal tension glaucoma. Surv Ophthalmol 2002;47(Suppl 1):S116-24. 